\title{
Using clinicopathological analysis of general practitioner skin surgery to determine educational requirements and guidelines
}

\author{
N H Cox, R Wagstaff, A W Popple
}

\begin{abstract}
Objective-To study the impact of skin surgery in general practice on the workload of a pathology laboratory and to identify what further training might be helpful.
\end{abstract}

Design-Analysis of skin biopsy specimens from general practitioners before and after their new contract to determine numbers of specimens, changes in diagnoses, adequacy of treatment of malignant tumours, and areas of low diagnostic accuracy.

Setting-District general hospital.

Subjects-All 1017 skin biopsy specimens from general practicc for 15 months before and 12 months after the new general practitioner contract.

Results-The number of pathology specimens received increased from 16 to 65 per month (median $=6$ submitted by each general practitioner in the post-contract year). The proportion of the more common pathological diagnoses was unchanged between the two periods, but the proportion of correctly diagnosed naevi, cysts, and seborrhoeic keratoses increased in the second. Although few diagnoses were overtly incorrect, accurate diagnosis of dermatofibromas and malignancies decreased after the contract, and the overall correct diagnosis rate for seborrhoeic keratoses, dermatofibromas, rashes, and malignancies was below $30 \%$. Only nine out of 21 squamous cell carcinomas were adequately excised with tumour free margins, and follow up of malignant tumours may have been inadequate.

Conclusions-Skin surgery in general practice has advantages but matters of concern are the increase in laboratory workload, the excision of some benign lesions, and the inappropriateness of biopsy of rashes. Squamous cell carcinoma and other malignant tumours submitted for pathological examination were often unsuspected and inadequately excised, and heightened suspicion is recommended. Pathology request forms may need redesigning to encourage provision of clinical details.

Cumberland Infirmary, Carlisle, CA2 7HY

N H Cox, MRCP, consultant dermatologist

$\mathrm{R}$ Wagstaff, $\mathrm{MB}$, registrar in public health medicine

A W Popple, FRCPaTh, consultant pathologist

Correspondence to:

Dr Cox.

This study was presented at the AGM of the British

Association of

Dermatologists, London,

July 1991

BMf 1992;304:93-6

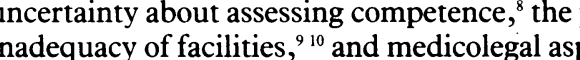

In East Cumbria health district, most general practitioners have performed minor surgery for many years. A recent survey (unpublished) showed that $90 \%$ of general practitioners intended to perform skin surgery after the new contract but that 59\% thought that further education would be helpful; many intended to perform procedures such as curettage for which they had never been trained. We were concerned that over a third of general practitioners intended to perform biopsies of rashes or blisters and over $10 \%$ intended to treat malignant melanoma or squamous cell carcinoma. To evaluate the effect of these intentions and to identify areas of educational need we analysed changes after the contract in the numbers of skin biopsy specimens submitted to the laboratory by general practitioners, the range of diagnoses, the accuracy of diagnosis of common disorders, and adequacy of surgery of tumours.

\section{Methods}

All skin biopsy specimens received by our pathology laboratory from general practitioners from 1 January 1989 ( 15 months before the contract) to 31 March 1991 (12 months after the contract) were analysed. The laboratory serves the whole of the district; few specimens are likely to be sent to other hospitals. From the biopsy forms we recorded the date of biopsy, adequacy of identification data (patient and general practitioner), appropriateness of tissue fixative, type of procedure (incision, excision, curettage, shave biopsy, other), clinical diagnosis or description, pathological diagnosis, and adequacy of excision in cases where this was important. We categorised diagnoses as naevi, cysts, viral warts, seborrhoeic keratoses, dermatofibromas, malignancies, rashes or blisters, and others. "Description" was used when a description but no presumptive diagnosis was given.

We carefully avoided being overcritical. Demographic details were considered adequate unless the name of the patient or general practitioner was omitted or illegible. Surgical excisions were coded as inadequate only when there was evidence of an incomplete excision of a malignant tumour. Clinicopathological correlation was coded after discussion between a dermatologist (NHC) and a pathologist (AWP), by using five categories of diagnostic accuracy (see appendix). For most results presented we have grouped together uncertain diagnoses, reasonable descriptions, and reasonable suggested diagnosis (categories 3-5).

All analyses were made using the statistical package for the social sciences and were carried out on precontract and postcontract data separately. When no difference was shown the combined results are cited.

Following analysis of preliminary results follow up details of malignancies were routinely requested from general practitioners by the laboratory, and registrations with departments of dermatology, radiotherapy, and plastic surgery were recorded as part of this study. Because several tumours had more than one specimen submitted in each part of the study period, and because 
adequacy of management was similar in each of these periods, the results of management of malignancies are presented for the study period as a whole.

\section{Results}

The number of specimens increased from 239 in the 15 months before the contract (mean 16/month) to 778 in the following 12 months (mean 65/month), an increase of $307 \%$ (table I). There was wide individual variation in the number of biopsy specimens submitted from 0 to 23 in the precontract period and from 0 to 45 in the postcontract period; the median numbers of specimens for the 90 general practitioners actually submitting samples ( $87 \%$ of the possible number) were three per general practitioner in the precontract period and six in the postcontract period. Only two submitted more than 25 specimens and nine more than 16 specimens in the postcontract year.

TABLE I-Pathological diagnosis of skin biopsy specimens in 15 months before and 12 months after the general practitioner contract. Results are numbers (percentages)

\begin{tabular}{|c|c|c|c|}
\hline Diagnosis & Before contract & After contract & $\begin{array}{l}\% \text { Increase } \\
\text { in No of } \\
\text { specimens } \\
\text { per month }\end{array}$ \\
\hline Naevus & $59(25)$ & $191(25)$ & 310 \\
\hline Seborrhoeic keratosis & $51(21)$ & $184(24)$ & 350 \\
\hline Cysts & $25(10)$ & $96(12)$ & 380 \\
\hline Dermatofibroma & $22(9)$ & $50(6)$ & 180 \\
\hline Malignancy & $18(8)$ & $38(5)$ & 160 \\
\hline Viral wart & $9(4)$ & $22(3)$ & 210 \\
\hline Rashes & 0 & $15(2)$ & - \\
\hline Other diagnoses & $51(21)$ & $173(22)$ & 320 \\
\hline Descriptive pathology & $4(2)$ & $9(1)$ & 125 \\
\hline Total & 239 & 778 & 310 \\
\hline
\end{tabular}

*This calculation takes into account the fact that the precontract period was 15 months and the postcontract period 12 months.

\section{DEMOGRAPHIC AND TECHNICAL FACTORS}

The number of forms with missing identifying data was constant (5\%) in both periods. An incorrect tissue fixative was used for at least $0.5 \%$ of specimens and was also constant throughout the study period. The frequency of specifying the type of procedure performed improved from $16 \%$ in the precontract period to $23 \%$ in the postcontract period. The age of the patient or date of birth was recorded in only $39 \%$ of cases.

\section{RANGE AND ACCURACY OF DIAGNOSES}

The proportion of lesions in each category of pathological diagnosis was unchanged between the two periods (table I).

Diagnostic accuracy in the two periods is shown in table II; categories 3-5 were combined. The overall correct diagnosis rates were $38 \%$ in the precontract period and $46 \%$ in the postcontract period, although fewer than $10 \%$ of specimens in most of the diagnosis groups were in category 2 (incorrect or inadequate clinical data). There was an increase in the proportions of correct diagnoses of naevi, seborrhoeic keratoses, and cysts after introduction of the contract but a decrease in the proportions of correct diagnoses of dermatofibromas, viral warts, and malignant tumours. Less than $30 \%$ of seborrhoeic keratoses, dermatofibromas, and malignant tumours were therefore accurately diagnosed. The most frequent incorrect diagnosis for seborrhoeic keratosis was mole or naevus $(21 \%)$ or wart $(8 \%)$, which were both considered reasonable suggestions, and a further $32 \%$ were coded as adequate descriptions. Dermatofibromas were more frequently diagnosed as naevi $(24 \%)$ than as the correct diagnosis $(19 \%)$, and cyst was also a frequent misdiagnosis $(12 \%)$ for these common lesions. The low frequency of correct diagnosis of rashes was rather more subjective as clinical details were usually descriptive and pathology reports rarely suggested a single diagnosis; only two patients with rashes were subsequently referred for a dermatological opinion and biopsy was not considered necessary in either.

\section{TUMOURS}

There were no changes in the adequacy of excision of tumours between the two study periods but diagnostic accuracy decreased.

Basal cell carcinoma-Of 21 specimens of basal cell carcinoma, only nine were considered to be possible malignancies (four diagnosed probable basal cell carcinoma, five as "? malignant"). Although primary excision was adequate in 15, both suspicion of malignancy and adequate excision occurred only in five. Only three of the six patients with inadequately excised tumours had been referred for a specialist opinion.

Malignant melanoma-None of the four cases of malignant melanoma was confidently diagnosed but one was described as suspicious. Other clinical diagnoses (one each) were naevus, "blistering mole with satellites," and haemangioma. All four were excised with margins free of tumour, but the recommendation by the reporting pathologist for a wider excision had apparently occurred in only three. A lentigo maligna was reasonably diagnosed as a naevus and adequately excised.

Squamous cell carcinoma-Twenty one specimens of squamous cell carcinoma were received from 16 patients, including two patients with two primary tumours each and one patient who had had two attempted excisions of a single lesion which were both incomplete. The only correct diagnosis was in a patient who had a recurrence of a squamous cell carcinoma which had been incompletely excised 18 months before the start of the study; only two further lesions were described as suspicious of malignancy. Primary excision was adequate in only eight of 18 new squamous cell carcinomas, although a further two specimens may have been just adequate (one was in incorrect fixative), and two adequately excised second attempt excisions were received. The combination of both suspicion of malignancy and adequate excision occurred in just one

TABLE II -Diagnostic accuracy^ before and after the contract. Results are numbers (percentages) of clinical diagnoses confirmed by the pathology specimens submitted

\begin{tabular}{|c|c|c|c|c|c|c|c|c|c|}
\hline \multirow{2}{*}{$\begin{array}{l}\text { Clinical } \\
\text { diagnosis }\end{array}$} & \multicolumn{4}{|c|}{ Before contract } & \multicolumn{4}{|c|}{ After contract } & \multirow{2}{*}{$\begin{array}{c}\text { Total } \\
\text { correct } \\
\text { diagnoses }\end{array}$} \\
\hline & Total & Category 1 & Category 2 & Category 3-5 & Total & Category 1 & Category 2 & Category 3-5 & \\
\hline Naevus & 59 & $34(58)$ & $3(5)$ & $22(37)$ & 191 & $124(65)$ & $9(5)$ & $55(30)$ & $158(64)$ \\
\hline Seborrhoeic keratosis & 51 & $8(16)$ & 0 & $43(84)$ & 184 & $59(32)$ & $6(3)$ & $119(65)$ & $67(29)$ \\
\hline Cysts & 25 & $14(54)$ & $9(37)$ & $2(8)$ & 96 & $85(88)$ & $10(10)$ & $2(2)$ & $99(81)$ \\
\hline Dermatofibroma & 22 & $7(32)$ & 0 & $15(68)$ & 50 & $7(14)$ & & $43(86)$ & $14(19)$ \\
\hline Malignancy & 18 & $8(44)$ & $1(6)$ & $9(50)$ & 38 & $5(13)$ & $3(8)$ & $30(79)$ & $13(23)$ \\
\hline Viral wart & 9 & $6(67)$ & $3(33)$ & 0 & 22 & $8(36)$ & $4(18)$ & $10(46)$ & $14(45)$ \\
\hline Rashes & 0 & & & & 15 & $4(27)$ & $1(7)$ & $10(66)$ & $4(27)$ \\
\hline Other diagnoses & 51 & $13(25)$ & $4(8)$ & $34(67)$ & 173 & $62(36)$ & $8(5)$ & $103(59)$ & $75(33)$ \\
\hline Total & 235 & $90(38)$ & $20(9)$ & $125(53)$ & 769 & $354(46)$ & $42(5)$ & $373(49)$ & $444(44)$ \\
\hline
\end{tabular}

^See appendix for categories of diagnostic accuracy. 
patient. Re-excision specimens were submitted for three patients (one after 12 months and one still inadequately excised), but only three of the remaining six patients with squamous cell carcinomas with definite incomplete excision had been referred for further surgery or radiotherapy locally; two were being followed up without further treatment and in one case no follow up information had been received.

\section{Discussion}

Our district is well suited to assess the impact of minor surgery in general practice because the district general hospital is geographically isolated and because most general practitioners performed some skin surgery before the new contract. The fourfold rise in the number of specimens submitted for pathological examination was greater than expected but similar to figures from other districts ${ }^{1213}$; one of these studies also reported no parallel decrease in specimens from the dermatology department. ${ }^{13}$ The proportion of inadequately identifiable or incorrectly fixed specimens remained constant, possibly because few general practitioners started performing skin surgery only after the contract. Similarly, the low frequency of recording the age of patients was constant in both parts of the study and was probably due to the fact that laboratory request forms, designed for use with hospital labels, did not have a separate section for recording age.

An important aim of our study was to identify aspects for further education. Those in favour of general practitioner skin surgery argue that there will be a learning curve until general practitioners realise their limitations. This argument actually provides strong justification for our study. The median number of biopsy specimens per general practitioner in the postcontract year (six specimens) was less than most dermatology trainees submit in each week of their supervised training, and the educational points emerging from our study of 1017 specimens could not be achieved by the "median general practitioner" in several working lifetimes.

The main areas of educational need were identified by assessing diagnostic accuracy and adequacy of treatment. Diagnostic categories were chosen to include naevi, cysts, and seborrhoeic keratoses (all high volume specimens); viral warts (an inappropriate lesion for treatment by excision); dermatofibromas (identified earlier as a diagnostic blind spot); rashes (for which an expert opinion is more appropriate than a biopsy); and malignant tumours (specimens of lower frequency but greater importance). The proportion of each of these groups was virtually identical in the two parts of the study.

The overall correct diagnosis rate was $44 \%$, similar to the $42 \%$ reported by Pitcher et al. ${ }^{13}$ Our study and those of others ${ }^{1314}$ were all based on pathology specimens; they do not therefore measure overall diagnostic accuracy because they exclude accurately diagnosed lesions treated by reassurance, surgery without pathological examination, or referral. Additionally, analysis of accuracy depends on the clinical information provided.

There were, however, some interesting findings. The proportion of correctly diagnosed naevi, cysts, and seborrhoeic keratoses increased after the new contract-as expected; these were likely to be lesions where general practitioners were most confident of the diagnosis. However, the proportion of correctly diagnosed tumours and dermatofibromas fell. Although most dermatofibromas were adequately described or had a reasonable suggested diagnosis, the correct diagnostic rate was only $19 \%$, fell after the contract, and was exceeded by the proportion diagnosed as naevi. Similarly, although wart, mole, horn, keratosis, or a description were all accepted as reasonable suggestions for a diagnosis of seborrhoeic keratosis, only $30 \%$ of these common lesions were correctly diagnosed (similar to the $35 \%$ rate for non-specialists cited in the study by Stern $\left.e t a l^{14}\right)$. Although we could have accepted wart alone and keratosis alone as correct diagnoses for seborrhoeic keratosis, only $8 \%$ were diagnosed as warts, and keratosis alone was used so loosely for actinic keratosis, seborrhoeic keratosis, keratoacanthoma, viral wart, and squamous cell carcinoma that we coded it as a description rather than a diagnosis. Some excisions are probably performed for diagnostic reasons when a specialist might either advise against excision or use a cosmetically superior treatment based on a firm diagnosis, and Shrank has questioned whether a patient's preference for surgery in the practice rather than at hospital can be genuine unless the option of reassurance without surgery is also considered.

Biopsy specimens of rashes received during the study did not appear to have given useful information as none had a single definite pathological diagnosis. Furthermore, none of these patients subsequently referred for a dermatological opinion were considered to have needed a biopsy for diagnosis, and the results were misleading in two cases. Because biopsy of rashes and blisters may require careful selection of the appropriate part of a rash, tissue for special techniques such as immunofluorescence, and greater knowledge of skin disease than can reasonably be expected of general practitioners, we advise that all rashes where biopsy is considered by the general practitioner should be referred.

Although it has been argued that general practice skin surgery will lead to early diagnosis of unsuspected melanoma, ${ }^{12}$ our results suggest problems in tumour management. Interestingly, the tumour which caused most concern was squamous cell carcinoma, of which only three out of 21 were suspected to be malignant. The only correctly diagnosed squamous cell carcinoma was one where a previous histology report 18 months before the study period had indicated inadequate excision. We received almost as many specimens of squamous cell carcinoma as of basal cell carcinoma, even though the incidence of squamous cell carcinoma in the United Kingdom is probably about tenfold less than that of basal cell carcinoma and twice as many general practitioners are prepared to treat basal cell carcinoma as would knowingly treat squamous cell carcinoma (unpublished findings). This diagnostic difficulty over squamous cell carcinoma was also recognised in a recent Australian study ${ }^{15}$ which found a correct diagnosis rate of $51 \%$ for dermatologists, $35 \%$ for surgeons, and $15 \%$ for general practitioners. The borders of a squamous cell carcinoma may be poorly demarcated and they often occur at difficult sites so there is a high risk of inadequate surgery if the diagnosis is not suspected. The high proportion of inadequate excisions is therefore likely to be more closely related to a low index of suspicion than to poor surgical skills. Although most squamous cell carcinomas seen in general practice are likely to be recognised as suspicious and referred for specialist clinical opinion, the combination of a relatively large volume of cases, low rate of diagnosis, and frequently inadequate surgery highlights squamous cell carcinomas as a subject for further education and emphasises the importance of not compromising surgical margins when excising malignancies.

Management of malignant melanoma caused less concern, possibly because of increased awareness of this tumour. Although only one of four nodular melanomas was suspected to be malignant, all four were excised with tumour free margins. Diagnosis of basal cell carcinoma also caused problems, but the 
adequacy of excision was better and the implications of an incompletely excised basal cell carcinoma are usually not as serious. Nevertheless, it is clear from our results that attention to pathology reports is critical as some tumours where recurrence is expected do not appear to have had further treatment. The follow up data on malignancies adds support to the view that adequate record keeping is a valid criterion to include in assessment of premises for minor surgery; this was the single most frequent reason for failure to meet criteria for adequacy of premises in a recent study. ${ }^{9}$

We conclude that minor surgery in general practice has advantages but also raises concern about the increase in laboratory workload, the likelihood that some benign lesions may be excised for no reason other than diagnostic uncertainty, the inappropriateness of biopsy of rashes, and the generally poor rate of diagnosis and adequate treatment of malignant tumours. Our results highlight the relatively small number of specimens submitted by even the most prolific general practitioners, and suggest that further training and guidelines may be helpful.

1 Department of Health and Welsh Office. General practice in the National Health Service: a new contract. London: Department of Health and W'elsh Office, 1989.

2 Brown JS. Minor operations in general practice. BMF 1979;i:1609-10.

3 Godfrey $\mathrm{E}$, Watkiss $M$, Schneiden $\mathrm{H}$. Initiation and evaluation of a pilo scheme for minor surgery in general practice. Health Trends 1990;22:57-9.

4 Sharman J. Patient's response to a general practice minor surgery service. Practitioner 1986;230:27-9.

5 Shrank AB. Correspondence. Health Trends 1990;22:181.

6 Slater DN. Screening and the 1990 contract. BMF 1991;300:1074.

7 Pringle M, Hasler J, De Marco P. Training for minor surgery in general practice during preregistration surgical posts. BMf 1991;302:830-2

8 Richards T. Competence for GP surgery. BMF 1991; 301:307-8.

9 Zoltie N, Hoult G. Adequacy of general practitioners' premises for minor Zoltie N, Hoult G. Adequacy of

10 Morgan DR, Lamont TJ, Dawson JD, Booth C. Decontamination of instruments and control of cross infection in general practice. $B M 7$ 1991;300:1379-80.

11 Dando P. Medico-legal aspects of minor surgery. Fournal of the Medical Defence Union 1991:1:12-3.

12. Hunt AC, Sherwood A. Competence for GP surgery. BM 1991;301:495

13 Pitcher R, Gould DJ, Bowers DW. An analysis of the effects of general practice minor surgery clinics on the workload of a district general hospital pathology and dermatology department. Brf Dermatol 1991:125(suppl 38):93.

14 Stern RS, Boudreux C, Arndt KA. Diagnostic accuracy and appropriateness of care for seborrheic keratoses. A pilot study of an approach to quality assurance for cutaneous surgery. FAMA 1991;265:74-7.

15 Nixon RDL, Dorevitch AP, Marks R. Squamous cell carcinoma of the skin accuracy of clinical diagnosis and outcome on follow-up in Australia. Med F A ust 1986;144:235-9.

(Accepted 9 October 1991)

\section{Appendix}

(1) Correct diagnosis-Accurate diagnoses were recorded; "mole" was taken to mean naevus.

(2) Incorrect diagnosis-Cases where (a) clinical information was omitted entirely or was brief and uninformative (such as "lump"), (b) the clinical diagnosis suggested was inconceivable given the pathological diagnosis (such as diagnosis of lipoma as a wart), or (c) the diagnosis was potentially dangerous - for example, "mole" for squamous cell carcinoma.

(3) Uncertain-For example, the clinical information for most rashes biopsied did not enable the accuracy of diagnosis to be established.

(4) Adequate description-For example, keratosis was used for a wide variety of different diagnoses and as a descriptive term, so was coded as a description unless qualified by a more specific term such as actinic (solar) keratosis, etc.

(5) Reasonable suggested diagnosis-Almost all pathological diagnoses where the clinical diagnosis was not accurate but suggested "mole" or "wart" were classified as category 5 unless the suggestion was entirely inappropriate. For analysis of tumours, we specifically recorded whether descriptions or suggested diagnoses included the possibility of any malignant disorder.

\title{
Diagnostic value of microtympanometry in primary care
}

\author{
Ruut A de Melker
}

\section{Abstract}

Objective-To determine the reliability, validity, and feasibility of a new hand held microtympanometer.

Design-Comparison of microtympanometry by two independent observations of a general practitioner and a nurse, and against a validated reference instrument.

Setting-Primary care health centre of a school for the deaf in the United States.

Subjects-111 schoolchildren receiving a regular check up.

Main outcome measures-Tympanometry with the Grason Stadler 28, classified with a slightly modified Jerger's classification.

Results-Interobserver reliability was $\mathbf{0 . 9 5}$ (Cohen's $x$ ). Results of microtympanometry were highly comparable with results of the reference instrument (likelihood ratio of positive results, 161·2).

Conclusions - The microtympanometer could be used in general practice: it is hand held, child friendly, easy to handle, and accurate.

Department of General Practice, University of Utrecht, 3511 ZC Utrecht, Netherlands

Ruut A de Melker, MD, professor

BMF 1992;304:96-8

\section{Introduction}

Tympanometry is a reliable and sensitive test for diagnosing otitis media with effusion in children, ${ }^{1-3}$ which is difficult to diagnose in general practice ${ }^{+6}$ : data collected by history is of limited value ${ }^{78}$ and otoscopy is rather unreliable. ${ }^{14910}$ The cumulative incidence of bilateral otitis media with effusion is $50-60 \%$ in open populations of children aged 2-6 years. ${ }^{6-811}$ About 5\% of 5 year olds have severe otitis media with effusion and suffer developmentally as a result. ${ }^{12-14}$

The hand held microtympanometer (Welch Allyn, Skaneateles Falls, New York) seems a good instrument to diagnose otitis media with effusion in primary care, but has not been sufficiently validated. ${ }^{15}$ The aim of this study is to determine the reliability, validity, and feasibility of using the microtympanometer. I set out to determine the interobserver and intraobserver reliability of microtympanometry in a group of schoolchildren aged 1-16 years; to discover the likelihood ratio of positive and negative results of the microtympanometer with the Grason Stadler GSI 28 tympanometer as reference instrument; and to assess the feasibility of a general practitioner and a nurse trained in ear, nose, and throat practice using the microtympanometer in a primary care setting.

\section{Subjects and methods}

The population consisted of 111 schoolchildren aged 1-16 years in a school for the deaf. They came for a regular ear, nose, and throat checkup in the primary care health centre of the school during the period November 1989 to January 1990. Primary health care was delivered by two nurses, a family doctor, and a ear, nose, and throat surgeon as consultant.

The history of upper respiratory tract infections was 